\title{
The Indigo Molecule Revisited Again: Assessment of the Minnesota Family of Density Functionals for the Prediction of Its Maximum Absorption Wavelengths in Various Solvents
}

\author{
Francisco Cervantes-Navarro and Daniel Glossman-Mitnik \\ NANOCOSMOS Virtual Lab, Centro de Investigación en Materiales Avanzados, Miguel de Cervantes 120, \\ Complejo Industrial Chihuahua, 31109 Chihuahua, CHIH, Mexico \\ Correspondence should be addressed to Daniel Glossman-Mitnik; daniel.glossman@cimav.edu.mx
}

Received 28 April 2012; Accepted 18 May 2012

Academic Editor: Cherumuttathu H. Suresh

Copyright (c) 2013 F. Cervantes-Navarro and D. Glossman-Mitnik. This is an open access article distributed under the Creative Commons Attribution License, which permits unrestricted use, distribution, and reproduction in any medium, provided the original work is properly cited.

The Minnesota family of density functionals (M05, M05-2X, M06, M06L, M06-2X, and M06-HF) were evaluated for the calculation of the UV-Vis spectra of the indigo molecule in solvents of different polarities using time-dependent density functional theory (TD-DFT) and the polarized continuum model (PCM). The maximum absorption wavelengths predicted for each functional were compared with the known experimental results.

\section{Introduction}

The indigo molecule and its derivatives are of fundamental importance in electrochemistry because they have potential applications in the field of electrochemical solar cells and dyesensitized solar cells. They can also be used in the electrochemical storage of energy, as metal corrosion inhibitors, and in the area of electrochemical fuel cells.

Density functional theory (DFT) [1], especially since the work of Kohn and Sham [2], is based entirely on the exchange and correlation density functional. Because this functional is not exactly known, it is necessary to use several approximations. The simplest approximation is called the local density approximation or LDA $[3,4]$. The next step was to propose several functional forms that were based not only on density but also on the density gradients to determine the generalized gradient approximation or GGA. One example of this type of functionals is the BLYP, which combines the B [5] exchange functional with the LYP $[6,7]$ correlation functional. Subsequently, it was discovered that one could better describe the density of a system and better predict its properties using an element of the Hartree-Fock (HF) or exact exchange. These new functionals are called hybrid GGA functionals, two examples of which are the ubiquitous B3LYP [5] and PBE0 [8-10] functionals. Later, it was recognized that these improvements could be compared to the process of climbing a ladder, with each rung leading to functionals that could be used to predict the experimental properties more accurately. This path has been called "Jacob's Ladder" [11]. The next step was to design functionals that, in addition to featuring the above-described properties, also were dependent on the kinetic energy density. These types of functionals are called meta-GGAs. When they included some degree of HF exchange, they were used to develop the hybrid meta-GGAs, the most modern functionals known. Using hybrid meta-GGAs has made it possible to calculate binding energies with chemical precision, that is, such that the predictions made are comparable to the experimental results. Included in this last family are the functionals developed at the University of Minnesota by Truhlar et al., known as M05, M05-2X, M06, M06L, M06-2X, and M06-HF [12-14]. Except for the local functional M06L, these functionals are hybrid meta-GGAs; they incorporate varying degrees of HF exchange, which were determined by adjusting their predictions to match the experimental values for several properties. However, because these functionals are approximations of the unknown exact energy density functional, the process of determining which functional should be used for a given 
system and the properties predicted by it requires one to perform calculations for each system and property and then compare them with the experimental results.

In computational chemistry and molecular modeling, research is conducted using what is called a "model chemistry." A model chemistry combines a density functional with a basis set, and when a solvent is included, the parameters for its simulation must also be considered. The indigo molecule presents a good opportunity to analyze the performance of a density functional and the prediction of its properties because the experimental UV-Vis spectra of indigo in different solvents have been empirically verified. The indigo molecule and its derivatives are of particular interest in electrochemistry because they have potential applications in electrochemical solar cells, dye-sensitized solar cells, electrochemical energy storage, as metal corrosion inhibitors, as components of fuel cells, and in artificial photosynthesis.

The aim of this study was thus to assess the performance of the Minnesota family of density functionals in predicting the maximum absorption wavelengths of indigo in several different solvents. These predictions will allow us to better understand not only which functional best describes this specific property in each particular system but also whether these density functionals behave differently in each case due to the different dipolar moments of each of the solvents.

\section{Theory and Computational Details}

All of the computational studies were performed with the Gaussian 09 [15] series of programs using the density functional methods implemented in the computational package. The equilibrium geometries of the molecules were determined using the gradient technique. The force constants and vibrational frequencies were determined by computing the analytical frequencies at the stationary points obtained after optimization to verify the presence of true minima. The basis sets used in this study were $6-311 \mathrm{G}(\mathrm{d}, \mathrm{p})$ and $6-311+\mathrm{G}(2 \mathrm{~d}, \mathrm{p})$, which are described elsewhere [16-19].

To calculate the molecular structure and properties of the systems studied, we chose the hybrid meta-GGA density functionals M05, M05-2X, M06, M06L, M06-2X, and M06HF [12-14], which consistently provide satisfactory results in terms of several structural and thermodynamic properties. The solvation energies were computed using the integral equation formalism-polarizable continuum model (IEF-PCM) [20], including the UAKS model. Dimethyl sulfoxide, ethanol, tetrachloroethane, chloroform, carbon tetrachloride, and benzene were used as the solvents.

The ultraviolet (UV-Vis) spectra of the studied systems were calculated by solving the time-dependent DFT (TDDFT) equations using the method implemented in Gaussian 09 [16, 21-23]. The equations were solved for 20 excited states. The infrared (IR) and ultraviolet (UV-Vis) spectra were calculated and visualized using the SWizard program [24, 25]. In all cases, the spectra displayed the calculated frequencies and absorption wavelengths, which allowed us to predict the maximum absorption wavelength of the studied system.

\section{Results and Discussion}

The optimized structure of the indigo molecule was calculated using the six density functionals in each of six solvents with different polarity values: dimethyl sulfoxide (DMSO; $\varepsilon=46.7)$, ethanol $(\varepsilon=24.6)$, tetrachloroethane (TCE; $\varepsilon=$ 8.2), chloroform $(\varepsilon=4.9)$, carbon tetrachloride $\left(\mathrm{CCl}_{4} ; \varepsilon=\right.$ $2.2)$, and benzene $(\varepsilon=2.2)$. In all cases, the frequencies and IR spectra obtained confirmed that these values were true minima. Based on the optimized ground-state molecular structures, we calculated the absorption spectra in each case in three steps:

(1) TD-DFT calculations to determine the vertical excitation for the ground state equilibrium geometry using the IEF-PCM solvation model, which corresponds to a linear response under nonequilibrium solvation.

(2) Ground state calculations to determine the energy in the IEF-PCM solvation model and to prepare the system for state-specific non-equilibrium solvation using the solvent reaction field from the ground state.

(3) The computation of the nonequilibrium solvation energy based on the ground state and the first excited state energy using the state-specific method; selfconsistent IEF-PCM calculations to compute the energy in solution by making the electrostatic potential of the solute self-consistent with the reaction field.

The computation of the nonequilibrium solvation energy based on the ground state and the first excited state energy using the state-specific method; self-consistent IEF-PCM calculations to compute the energy in solution by making the electrostatic potential of the solute self-consistent with the reaction field.

The results of the calculations are presented in Table 1, which shows the maximum wavelength absorption (in $\mathrm{nm}$ ) for the indigo molecule in solvents with decreasing polarity and for each of the density functionals considered. The experimental values are those reported by Jacquemin et al. [26]. Also, we are including a table (Table 2) showing the same results but utilizing absorption energy values (in $\mathrm{eV}$ ), because the energies are more intuitive than wavelengths when comparing energy accuracies.

The results listed in Tables 1 and 2 clearly indicate that for solvents with high dipolar moment (DMSO and ethanol), the M05 and M06 functionals predict the maximum absorption wavelength (or the absorption energy) of the indigo molecule much more accurately than the other functionals given the experimental results. In particular, the performance of these functionals in connection with ethanol is impressive. For a medium-polarity solvent such as TCE, the M05 and M06 density functionals are also preferable to the others, although the accuracy is lower. When used with the low-dipolemoment solvents chloroform, $\mathrm{CCl}_{4}$ and benzene, none of the functionals accurately predict the maximum absorption wavelength of indigo; although M05, M06, and M06L provide good approximate values, the first two underestimate the value, and the last one overestimates it. There is no case in which the functionals with high HF exchange content are 
TABLE 1: Maximum absorption wavelengths of the indigo molecule (in $\mathrm{nm}$ ) calculated using the Minnesota density functionals in several solvents.

\begin{tabular}{|c|c|c|c|c|c|c|c|}
\hline Solvent & M05 & M05-2X & M06 & M06L & M06-2X & M06-HF & Exp. \\
\hline DMSO & 614 & 562 & 610 & 667 & 565 & 518 & $620 \pm 1$ \\
\hline Ethanol & 606 & 556 & 606 & 662 & 559 & 514 & $608 \pm 2$ \\
\hline TCE & 597 & 546 & 593 & 652 & 549 & 503 & $613 \pm 8$ \\
\hline Chloroform & 585 & 535 & 581 & 637 & 538 & 493 & $605 \pm 3$ \\
\hline $\mathrm{CCl}_{4}$ & 565 & 535 & 581 & 637 & 538 & 493 & $605 \pm 3$ \\
\hline Benzene & 565 & 518 & 562 & 617 & 518 & 476 & $595 \pm 5$ \\
\hline
\end{tabular}

TABLE 2: Maximum absorption energies of the indigo molecule (in eV) calculated using the Minnesota density functionals in several solvents.

\begin{tabular}{|c|c|c|c|c|c|c|c|}
\hline Solvent & M05 & M05-2X & M06 & M06L & M06-2X & M06-HF & Exp. \\
\hline DMSO & 2.02 & 2.21 & 2.03 & 1.86 & 2.19 & 2.39 & 2.00 \\
\hline Ethanol & 2.05 & 2.30 & 2.05 & 1.87 & 2.22 & 2.41 & 2.04 \\
\hline TCE & 2.08 & 2.27 & 2.09 & 1.90 & 2.26 & 2.47 & 2.02 \\
\hline Chloroform & 2.12 & 2.32 & 2.13 & 1.95 & 2.31 & 2.51 & 2.05 \\
\hline $\mathrm{CCl}_{4}$ & 2.19 & 2.32 & 2.13 & 1.95 & 2.31 & 2.51 & 2.05 \\
\hline Benzene & 2.19 & 2.39 & 2.21 & 2.01 & 2.39 & 2.60 & 2.08 \\
\hline
\end{tabular}

sufficiently accurate; rather, they clearly underestimate the experimental results.

Usually, when comparing the results of different functionals for the prediction of a given property (being all the other components of the model chemistry the same, i.e., the basis set, the model used to simulate the solvent, etc.), it is considered that the functional form and its parametrization are important points to be taken into account. However, the functionals used in this study have all the same functional form and have been parametrized with the same physical constants. It is here where our study acquires more relevance the only difference between the functionals is the inclusion of exact $\mathrm{HF}$ exchange in different percentages. Thus, the good results for the local functionals or for the functionals with lesser amounts of exact HF exchange, can be directly attributed to the fact that, at least for the systems used in this study, the inclusion of HF exact exchange is perjudicial for the prediction of the maximum absorption wavelengths.

\section{Conclusions}

The Minnesota family of density functionals were assessed to determine their performance in predicting the maximum absorption wavelength (or the absorption energies) of the indigo molecule in solvents with decreasing polarity. The results indicate that the M05 and M06 functionals provide relatively better calculations for solvents with high dipole moment such as DMSO and ethanol. For solvents with medium dipole moment such as TCE, the same functionals are better than the others, although they generate less accurate results than when used with the more polar solvents. Finally, for solvents with low dipole moment such as chloroform, $\mathrm{CCl}_{4}$, and benzene, the functionals M05, M06, and M06L yielded fairly accurate maximum wavelength values, with underestimates and overestimates of $20 \mathrm{~nm}$ on average.
Clearly, the inclusion of more HF exchange, as in the M052X, M06-2X, and M06-HF functionals, leads to inaccurate or underestimated values.

The results of this study demonstrate that the performance of these functionals not only is related to the system under study and the basis set considered but also depends on the solvent in which the molecule is immersed. These findings can help make it possible to select the most suitable functional in studying related indigoid molecules.

\section{Acknowledgments}

This work was partially supported by the Consejo Nacional de Ciencia y Tecnología (CONACYT, Mexico). F. CervantesNavarro gratefully acknowledges a fellowship from CONACYT. D. Glossman-Mitnik is a researcher with CONACYT and CIMAV.

\section{References}

[1] R. G. Parr and W. Yang, Density Functional Theory of Atoms and Molecules, Oxford University Press, New York, NY, USA, 1989.

[2] W. Kohn and L. Sham, "Self-consistent equations including exchange and correlation effects," Physical Review, vol. 140, pp. A1133-A1138, 1965.

[3] J. Slater, The Self-Consistent Field for Molecules and Solids, McGraw-Hill, New York, NY, USA, 1974.

[4] S. Vosko, L. Wilk, and M. Nusair, "Accurate spin-dependent electron liquid correlation energies for local spin density calculations: a critical analysis," Canadian Journal of Physics, vol. 58, no. 8, pp. 1200-1211, 1980.

[5] A. D. Becke, "Density-functional exchange-energy approximation with correct asymptotic-behavior," Physical Review A, vol. 38, no. 6, pp. 3098-3100, 1988.

[6] C. Lee, W. Yang, and R. G. Parr, "Development of the colle-salvetti correlation-energy formula into a functional of 
the electron-density," Physical Review B, vol. 37, no. 2, pp. 785-789, 1988.

[7] B. Miehlich, A. Savin, H. Stoll, and H. Preuss, "Results obtained with the correlation energy density functionals of becke and Lee, Yang and Parr," Chemical Physics Letters, vol. 157, no. 3, pp. 200-206, 1989.

[8] J. P. Perdew, K. Burke, and M. Ernzerhof, "Generalized gradient approximation made simple," Physical Review Letters, vol. 77, no. 18, pp. 3865-3868, 1996.

[9] J. Perdew, K. Burke, and M. Enzerhof, "Generalized gradient approximation made simple," Physical Review Letters, vol. 78, pp. 1396-1396, 1997.

[10] C. Adamo and V. Barone, "Toward reliable density functional methods without adjustable parameters: the PBE0 model," Journal of Chemical Physics, vol. 110, no. 13, pp. 6158-6170, 1999.

[11] J. P. Perdew, A. Ruzsinszky, J. Tao, V. N. Staroverov, G. E. Scuseria, and G. I. Csonka, "Prescription for the design and selection of density functional approximations: more constraint satisfaction with fewer fits," Journal of Chemical Physics, vol. 123, no. 6, Article ID 062201, 2005.

[12] Y. Zhao and D. G. Truhlar, "Density functionals with broad applicability in chemistry," Accounts of Chemical Research, vol. 41, no. 2, pp. 157-167, 2008.

[13] Y. Zhao and D. G. Truhlar, "The M06 suite of density functionals for main group thermochemistry, thermochemical kinetics, noncovalent interactions, excited states, and transition elements: two new functionals and systematic testing of four M06-class functionals and 12 other functionals," Theoretical Chemistry Accounts, vol. 120, no. 1-3, pp. 215-241, 2008.

[14] Y. Zhao and D. G. Truhlar, "Applications and validations of the Minnesota density functionals," Chemical Physics Letters, vol. 502, no. 1-3, pp. 1-13, 2011.

[15] M. J. Frisch, G. W. Trucks, H. B. Schlegel et al., Gaussian 09 Revision A.1, Gaussian, Wallingford, UK, 2009.

[16] E. Lewars, Computational Chemistry-Introduction to the Theory and Applications of Molecular and Quantum Mechanics, Kluwer Academic Publishers, Dordrecht, The Netherlands, 2003.

[17] D. C. Young, Computational Chemistry-A Practical Guide for Applying Techniques to Real-World Problems, John Wiley \& Sons, New York, NY, USA, 2001.

[18] F. Jensen, Introduction to Computational Chemistry, John Wiley \& Sons, Chichester, UK, 2nd edition, 2007.

[19] C. J. Cramer, Essentials of Computational Chemistry-Theories and Models, John Wiley \& Sons, Chichester, UK, 2nd edition, 2004.

[20] J. Tomasi, B. Mennucci, and E. Cancès, "The IEF version of the PCM solvation method: an overview of a new method addressed to study molecular solutes at the QM ab initio level," Journal of Molecular Structure, vol. 464, p. 211, 1999.

[21] R. E. Stratmann, G. E. Scuseria, and M. J. Frisch, "An efficient implementation of time-dependent density-functional theory for the calculation of excitation energies of large molecules," Journal of Chemical Physics, vol. 109, no. 19, pp. 8218-8224, 1998.

[22] R. Bauernschmitt and R. Ahlrichs, "Treatment of electronic excitations within the adiabatic approximation of time dependent density functional theory," Chemical Physics Letters, vol. 256, pp. 454-464, 1996.
[23] M. E. Casida, C. Jamorski, K. C. Casida, and D. R. Salahub, "Molecular excitation energies to high-lying bound states from time-dependent density-functional response theory: characterization and correction of the time-dependent local density approximation ionization threshold," Journal of Chemical Physics, vol. 108, no. 11, pp. 4439-4449, 1998.

[24] S. Gorelsky, "SWizard Program Revision 4.6".

[25] S. I. Gorelsky and A. B. P. Lever, "Electronic structure and spectra of ruthenium diimine complexes by density functional theory and INDO/S. Comparison of the two methods," Journal of Organometallic Chemistry, vol. 635, pp. 187-196, 2001.

[26] D. Jacquemin, J. Preat, V. Wathelet, and E. Perpete, "Substitution and chemical environment effects on the absorption spectrum of indigo," Journal of Chemical Physics, vol. 124, no. 7, 12 pages, 2006. 

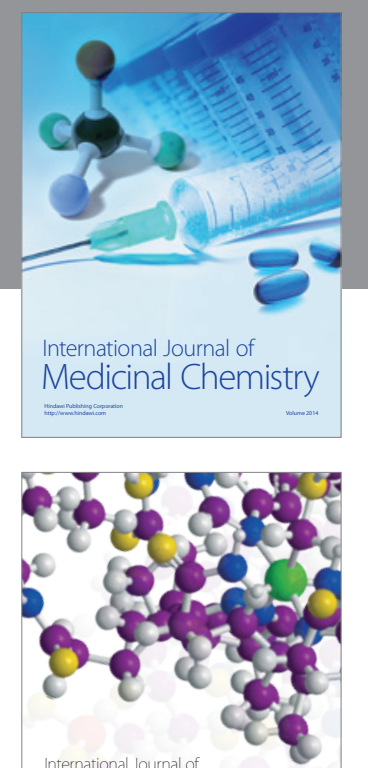

\section{Carbohydrate} Chemistry

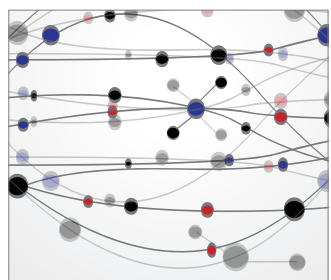

The Scientific World Journal
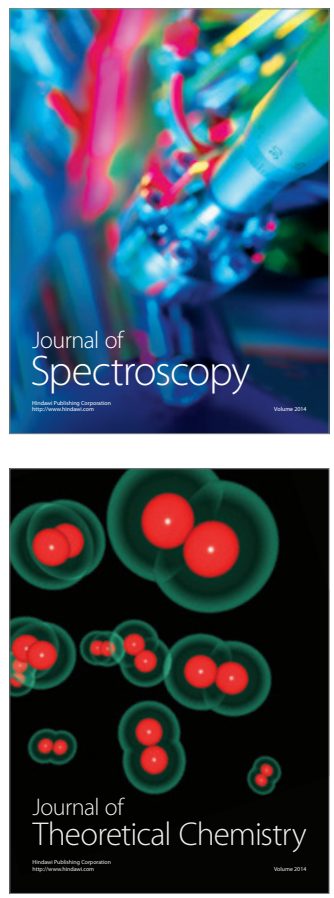
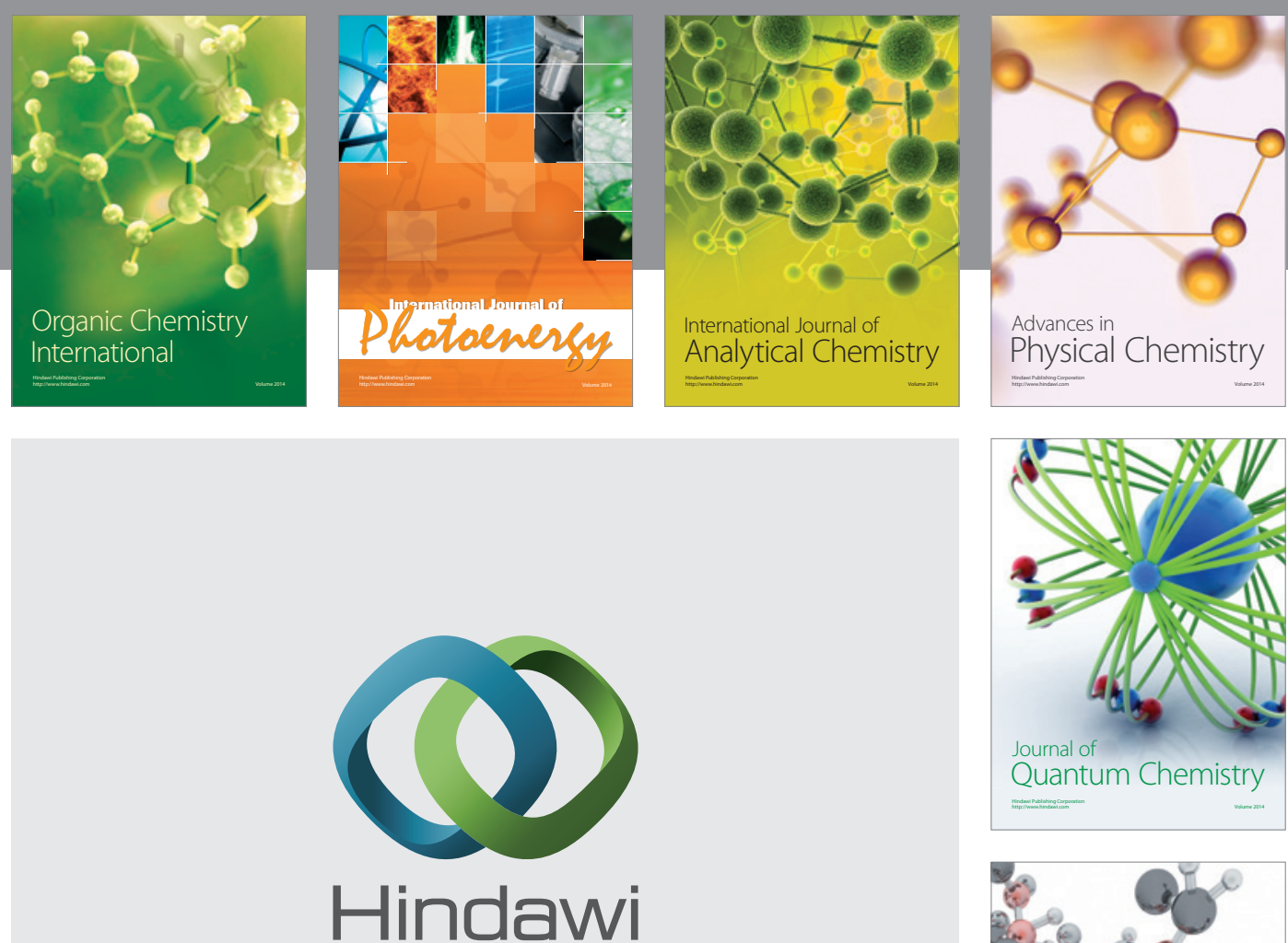

Submit your manuscripts at

http://www.hindawi.com

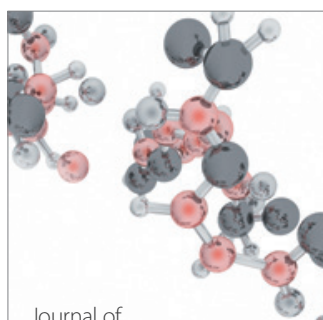

Analytical Methods

in Chemistry

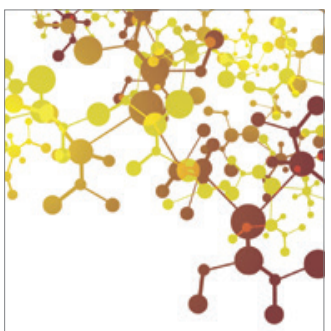

Journal of

Applied Chemistry

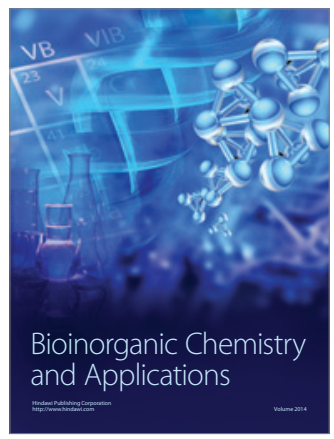

Inorganic Chemistry
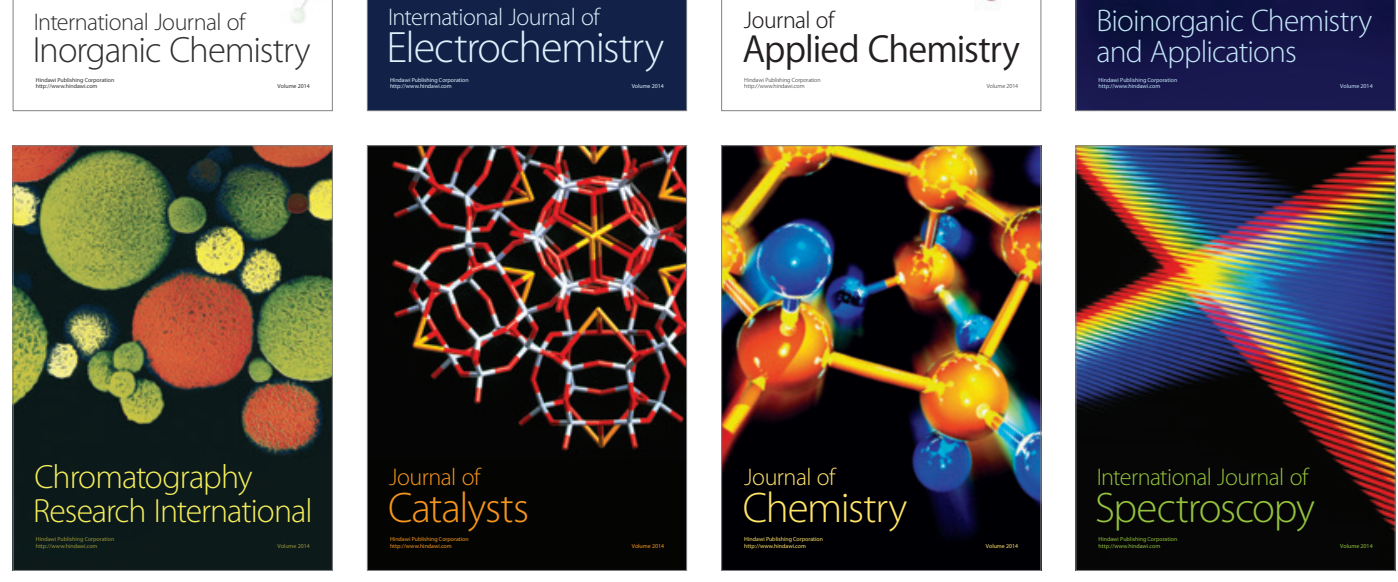